\title{
Moving Vortex States Studied by Current Flow in Single Crystal $\mathrm{Bi}_{2} \mathrm{Sr}_{2} \mathrm{CaCu}_{2} \mathrm{O}_{8+\delta}$
}

\author{
J. Mirković,* K. Kimura, and K. Kadowaki \\ CREST, Japan Science and Technology Corporation (JST) and Institute of Materials Science, \\ The University of Tsukuba, 1-1-1 Tennodai, Tsukuba 305-8573, Japan
}

(Received 18 May 1998)

\begin{abstract}
Nonequilibrium vortex states have been investigated in single crystalline $\mathrm{Bi}_{2} \mathrm{Sr}_{2} \mathrm{CaCu}_{2} \mathrm{O}_{8+\delta}$ using in-plane resistance and magnetization measurements. It was found that the first-order vortex-lattice melting transition (VLMT) phase line persists even in the dynamic vortex states in a wide range of driving forces and sharply separates the vortex solid and the vortex liquid phases. The activation energy $U$ deduced from the relation $R=R_{0} \exp \left(-U / k_{B} T\right)$ abruptly increases below VLMT, strongly depends on transport currents, and weakly depends on magnetic fields in the vortex solid state. The resistivity attains zero value sharply at VLMT in the low current limit. [S0031-9007(99)08681-0]

PACS numbers: 74.60.Ge, 74.60.Ec, 74.72.Hs
\end{abstract}

Despite many experimental and theoretical efforts in studying the intriguing physical properties of the vortex state, a full description of vortex matter in high temperature superconductors is far from being achieved. In particular, it remains unclear what happens in vortex matter under nonequilibrium dynamic conditions as in the presence of high driving forces by flowing currents. Some theoretical [1,2] and experimental studies done in the layered superconductors $2 \mathrm{H}-\mathrm{NbSe}_{2}[3,4]$ and $\mathrm{YBa}_{2} \mathrm{Cu}_{3} \mathrm{O}_{7-\delta}$ [5] suggested a peculiar behavior of moving vortex lattice with novel dynamic phase transitions and regimes like plastic and elastic motion of vortex crystal, but little is known regarding dynamic vortex states in $\mathrm{Bi}_{2} \mathrm{Sr}_{2} \mathrm{CaCu}_{2} \mathrm{O}_{8+\delta}$. Low magnetic fields and a very low level of resistivity where the occurrence of the vortex phase transition is expected, as well as structural inhomogeneities of samples, make the first-order vortex-lattice melting transition (VLMT) more difficult to be observed by steady current flow in $\mathrm{Bi}_{2} \mathrm{Sr}_{2} \mathrm{CaCu}_{2} \mathrm{O}_{8+\delta}$ single crystals [6-11] than in $\mathrm{YBa}_{2} \mathrm{Cu}_{3} \mathrm{O}_{7-\delta}[5,12,13]$.

With the final goal of obtaining further insight into the dynamic vortex states in $\mathrm{Bi}_{2} \mathrm{Sr}_{2} \mathrm{CaCu}_{2} \mathrm{O}_{8+\delta}$ and their relations with VLMT in the static case, we performed the high sensitive in-plane resistance measurements in $\mathrm{Bi}_{2} \mathrm{Sr}_{2} \mathrm{CaCu}_{2} \mathrm{O}_{8+\delta}$ single crystal as a function of temperature in magnetic fields applied along the $c$ axis with a wide range of current levels. Moving vortex state was measured at low levels of the resistance, several orders of magnitude below $R\left(T_{m}\right)$ where VLMT occurs. It was observed that the VLMT persists even in the moving lattice in a wide range of driving force and sharply separates phases, the vortex liquid and the vortex solid. Therefore, the main subject of this Letter is the comparative study of vortex dynamics in the vortex solid and the vortex liquid state near VLMT. In contrast to liquid, we show the sharp increase of the activation barrier in the vortex solid state as the driving current decreases implying the tendency that the melting and the zero resistivity transition converge to a single transition in the zero current limit.
The single crystal of $\mathrm{Bi}_{2} \mathrm{Sr}_{2} \mathrm{CaCu}_{2} \mathrm{O}_{8+\delta}$ was selected from pieces of the platelike as-grown single crystals [14]. The superconducting transition in zero magnetic field was observed to be at $T_{c}=83.0 \mathrm{~K}$ with $\Delta T((90 \%-$ $\left.10 \%) \rho_{n}\right) \approx 1 \mathrm{~K}$, which indicates that this sample lies at a slightly overdoped region. The quality of the crystal was checked by various techniques such as ac-susceptibility, $\mathrm{x}$-ray rocking curve analyses, and angular dependence of resistance. The crystal was cut to the dimension of $2.7 \times 1.7 \times 0.01 \mathrm{~mm}^{3}$. Four electrical contacts for transport measurements were attached to the top of the surface of the crystal using $\mathrm{Ag}$ epoxy (contact resistance was less than $1 \Omega$ ). The in-plane resistance of $\mathrm{Bi}_{2} \mathrm{Sr}_{2} \mathrm{CaCu}_{2} \mathrm{O}_{8+\delta}$ was measured by the conventional four probe ac method with a narrow band lock-in technique at a low frequency of $16 \mathrm{~Hz}$. The measurements of resistance $\rho(H, T)$ were carried out as functions of temperature, current, and magnetic field, which was applied along the $c$ axis. The sample was cooled in fields and measured while it was being heated up; the temperature was slowly swept at a constant rate of $0.2 \mathrm{~K} / \mathrm{min}$. The magnetization $M(H, T)$ was detected with a commercial SQUID magnetometer (Quantum Design, MPMS-5S) with RSO (Reciprocal Sample Option).

Figure 1 shows the set of resistance curves as a function of temperature obtained in various magnetic fields below 800 Oe with a driving current of $10 \mathrm{~mA}$. At the low level of resistance, the $R(T)$ curves demonstrated a typical clear drop associated with VLMT (the temperature derivative of the resistance $d R / d T$ for a particular applied field of 246 Oe shown in the inset of Fig. 1 illustrates the sharpness of the resistance anomaly). Since resistivity is not thermodynamic physical quantity we also measured the isothermal magnetization on the same sample as shown previously $[8,9]$ and confirmed the phase transition as presented in the lower inset of Fig. 1 [entropy change at $T_{m}$ in the region 50$70 \mathrm{~K}$ was $\Delta s \approx\left(0.6 k_{B} /\right.$ vortex $) /$ layer, what is close to the value reported earlier [15]]. 


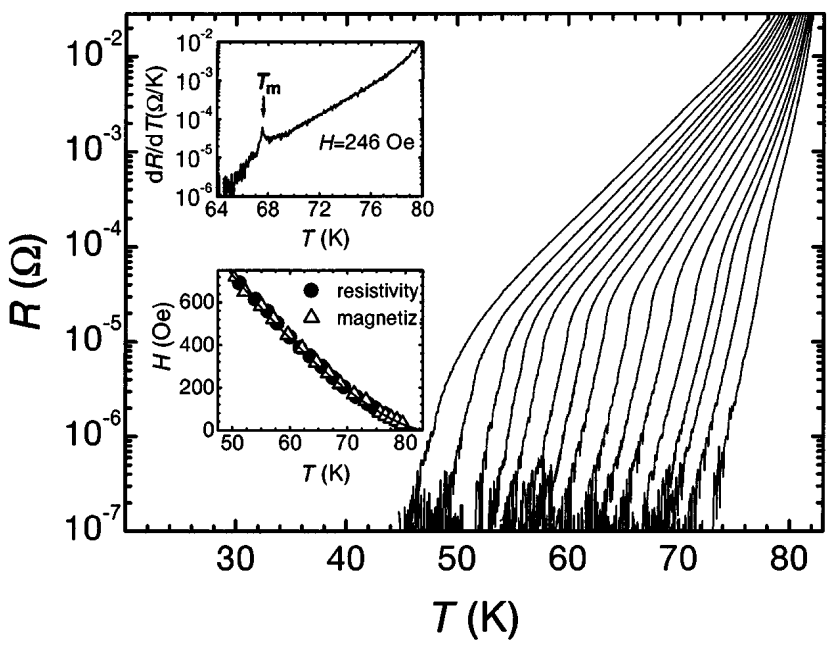

FIG. 1. Logarithmic plot of the in-plane resistance as a function of $T$ measured with the current of $10 \mathrm{~mA}$ in magnetic fields of (from right) 47, 75, 103, 123, 156, 201, 246, 299, 347, 388, 437, 502, 558, 614, 690, and 798 Oe. Upper inset: the arrow shows VLMT temperature $T_{m}$ defined from the peak of the temperature derivative of resistance $d R / d T$. Lower inset: The first-order $\mathrm{H}-\mathrm{T}$ phase diagram of $\mathrm{Bi}_{2} \mathrm{Sr}_{2} \mathrm{CaCu}_{2} \mathrm{O}_{8+\delta}$ obtained from both resistivity and magnetization measurements on the same sample. The solid line is a fitted curve for the resistivity data $H=H_{0}\left(1-T / T_{c}\right)^{\nu}$, where $H_{0}=2800 \mathrm{Oe}$ and $\nu=1.46$.

In order to better understand the vortex states in nonequilibrium conditions, we studied the current dependence of the melting transition in more detail. Figure 2 shows the set of $R(T)$ curves in the magnetic field of $H=200$ Oe obtained with different current density levels, from $I=1.5 \mathrm{~mA}$ up to $I=50 \mathrm{~mA}$. All curves $R(T)$ have a distinct single drop at $T_{m}=69.5 \mathrm{~K}$, which corresponds to the VLMT. The intensity of the signature of VLMT strongly depends on the current level $I$ : the resistance drop at $T_{m}$ becomes weaker as the current $I$ is raised, but a tiny anomaly can still be detectable at the highest currents $(I \simeq 50 \mathrm{~mA})$. The remarkable phenomenon is that the VLMT occurs even in the presence of such high driving forces (Fig. 2 inset). This means that the flowing lattice also melts. However, it is likely that the clear resistance signature of VLMT and difference between vortex liquid and vortex solid phase would be lost for currents higher than a certain threshold level of driving force, regardless of whether bulk pinning, surface barriers, or something else governs the transport properties. Further, $T_{m}$ demonstrates surprisingly weak transport current dependence. The increase of driving current density from $I=1.5 \mathrm{~mA}$ to $I=50 \mathrm{~mA}$ in a magnetic field of 200 Oe caused the shift of transition temperature only by $\Delta T \approx 0.3 \mathrm{~K}$ to lower temperatures. This experimental fact means that the dynamic system with moving vortices has a transition temperature $T_{m}$ very close to the one of the equilibrium, static vortex system as the intrinsic physical phenomenon (for a comparison, the Fig. 2 inset

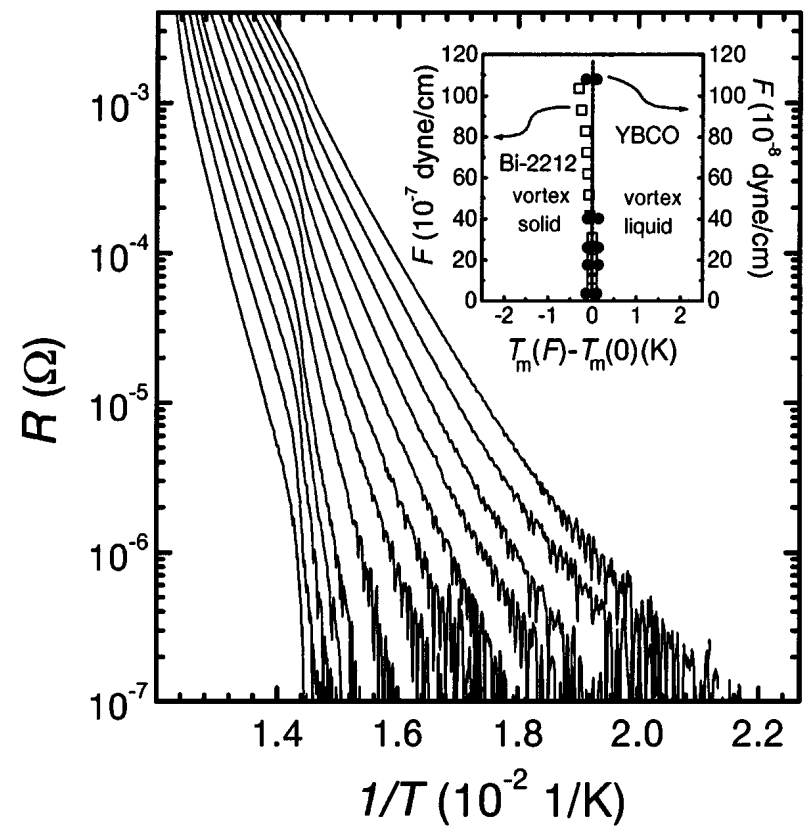

FIG. 2. Temperature dependence of the resistance in a fixed magnetic field of 200 Oe measured with various current density levels; (from left) $J=1.5,3,5,7.5,10,15,20,25,30,35$, 40, 45, and $50 \mathrm{~mA}$. Inset: Comparison of the dynamic phase diagrams for $\mathrm{Bi}_{2} \mathrm{Sr}_{2} \mathrm{CaCu}_{2} \mathrm{O}_{8+\delta}$ (squares) and $\mathrm{YBa}_{2} \mathrm{Cu}_{3} \mathrm{O}_{7-\delta}$ (circles) [5] ( $F$ denotes the driving force/unit length on a single vortex).

shows also data for the single crystal $\left.\mathrm{YBa}_{2} \mathrm{Cu}_{3} \mathrm{O}_{7-\delta}[5]\right)$. The certain although rather small shift of VLMT to lower temperature as driving forces are raised might be caused by the increased number of the current-driven defects in the vortex lattice, which in turn may support the fact that the vortex solid melts easier (alternatively, the same may happen when current affects interlayer Josephson coupling). Since, as is known, the vortex lattice melts like ice, one may find that the observed freezing of moving vortex lattice is reminiscent of the freezing of a river.

The next experimental finding which we point out is the current dependence of resistance around the VLMT. The temperature dependence of resistance demonstrated the qualitatively different behavior below and above VLMT. Below $T_{m}$, the slope of the long tail of $R(T)$ strongly depends on the current level, whereas above $T_{m}$, the resistance curves obtained by different current levels are weakly affected by $I$. We should stress that the resistance in the vortex solid phase with higher driving forces is very close to the value of it in the liquid phase. On the other hand, at the lower current levels the resistance drops sharply to zero value at a temperature very close to $T_{m}$ : it is likely that in the zero current limit, the zero resistivity transition temperature $T(\rho=0)$ coincides with the VLMT temperature as is indicated in the upper inset of Fig. 3. The same conclusion that the VLMT represents a boundary line of the true superconducting phase with vanishing linear resistivity for $I \rightarrow 0$ could be 


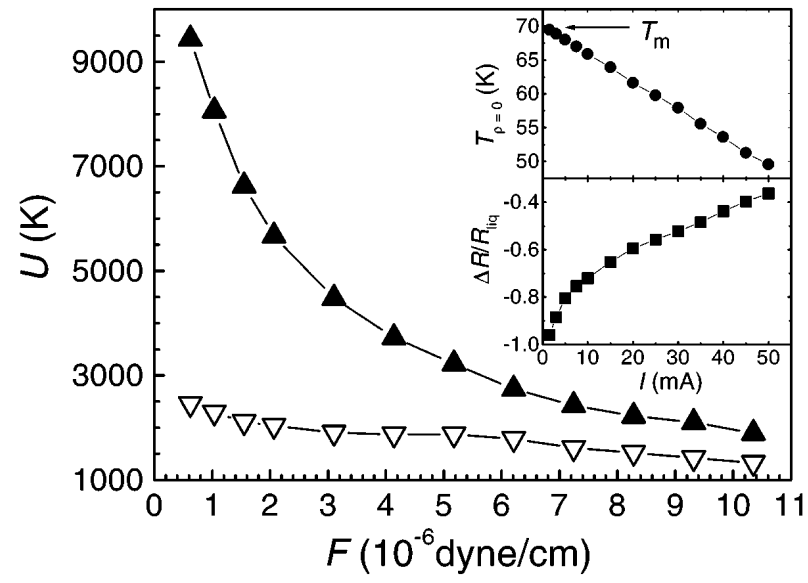

FIG. 3. Dependence of activation energy $U$ on the driving force/unit length $F$ on a single vortex as derived from the resistance measurements: black and unfilled triangles correspond to $U$ derived for the temperature $T=68.94 \mathrm{~K}$ (solid) and $T=71.7 \mathrm{~K}$ (liquid), respectively. The upper inset shows the current dependence of the zero resistivity transition temperature derived under the criterion of $R=$ $3.96 \times 10^{-7} \Omega$. The lower inset demonstrates the current dependence of $\delta R / R_{\text {liq }}$, where $\delta R=R_{\text {liq }}-R_{\text {sld }}$, with $R_{\text {liq }}$ and $R_{\text {sld }}$ resistance values at temperatures of 70.26 and $69.19 \mathrm{~K}$, respectively.

drawn from the force dependence of the activation energy $U$. Since the resistance demonstrated thermally activated behavior in rather wide ranges of temperature, we applied the relation $R(T)=R_{0} \exp \left(-U / k_{B} T\right)$ (where $k_{B}$ is the Boltzman constant) to our resistivity data obtained at the constant magnetic field of 200 Oe and estimated the activation potential $U$ directly from the slope of $\ln R$ versus $1 / T$ [16]. As is shown in Fig. 3, there is a sharp quantitative and qualitative difference in the dependence of $U$ on the driving force below and above VLMT. While the estimated $U$ demonstrates weak current dependence in the liquid phase, $U$ seems to have a tendency to grow and diverge in the solid phase as the current is decreased to the lower levels. The activation energy $U$ follows a powerlaw behavior: $U(I) \sim I^{\alpha}$, where $\alpha \approx-0.7$ for higher currents and $\alpha \approx-0.45$ in the low current region, in nice agreement with the Bragg-glass phase model [17]. The similar coefficient $\alpha \approx-0.6$, as well as the value of the activation energy, was previously reported from the measurements of relaxation of the remanent magnetization in the high temperature region [18] which, according to the weak collective pinning theory [19], authors related to the regime of the large bundles. On the other hand, the weak field dependence and rather high value of activation energy may correspond to the surface barriers [20,21]. However, although surface barriers definitely affect the transport properties and represent the relevant factor determining the activation energy in $\mathrm{Bi}_{2} \mathrm{Sr}_{2} \mathrm{CaCu}_{2} \mathrm{O}_{8+\delta}$ in both vortex solid and liquid phases [22], our recent experimental finding [23] suggested that, nevertheless, the surface barriers do not play a major role but act as a secondary effect in the wide range of fields and driving forces, at least for the samples studied and presented here [24].

In contrast to some predictions $[1,2]$ and experimental observations in other system $[3,4]$ we did not find the peculiar behavior such as plastic-elastic dynamic transition or any other dramatic change in behavior of the resistance within the measured range of driving current levels. Moreover, we also did not observe any signature of the pronounced peak effect which sometimes occurs as a precursor of VLMT, as it was observed in $\mathrm{YBa}_{2} \mathrm{Cu}_{3} \mathrm{O}_{7-\delta}$ systems and $\mathrm{Bi}_{2} \mathrm{Sr}_{2} \mathrm{CaCu}_{2} \mathrm{O}_{8+\delta}$ with columnar defects. Instead of that, the resistance changes quite smoothly by increasing the driving force in the solid phase. As an illustration, the relative difference of resistance between liquid and solid vortex states is presented in the lower inset of Fig. 3. The ratio $\Delta R / R_{\text {liq }}$, where $\Delta R=R_{\text {sld }}-R_{\text {liq }}$, and $R_{\text {sld }}, R_{\text {liq }}$ denote the resistance at temperatures of $69.19 \mathrm{~K}$ (solid) and $70.26 \mathrm{~K}$ (liquid), respectively, changes gradually with current density: almost linear current dependence was observed down to the lowest current density levels where $\Delta R$ converges more sharply towards the value of -1 . The observed behavior indicates a rather low pinning force in $\mathrm{Bi}_{2} \mathrm{Sr}_{2} \mathrm{CaCu}_{2} \mathrm{O}_{8+\delta}$ at high temperatures in accordance with the already noted 3D collectively pinned state from the current dependence of the activation energy $U(I)$. As is known, the tilt and shear energies in the mentioned regime dominate over pinning energy $[18,19]$.

In order to show the qualitative difference of dynamic response in two vortex phases in various magnetic fields, the set of resistance curves from Fig. 1 is replotted as a function of $T-T_{m}$ distance from VLMT. It is clearly seen in Fig. 4(a) that the resistance data below the VLMT
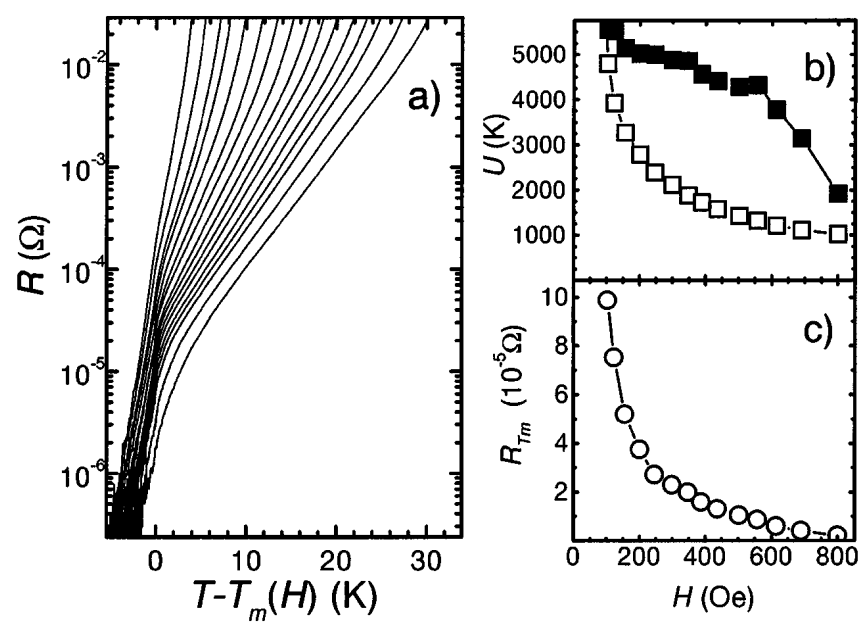

FIG. 4. (a) Set of resistance curves from Fig. 1 replotted as a function of $T-T_{m}$. (b) Field dependence of activation energy $U$ : Black squares correspond to the resistance level of $R=$ $1.19 \times 10^{-6} \Omega$ (vortex solid); unfilled squares correspond to the resistance level $R=1.7 \times 10^{-4} \Omega$ (vortex liquid). (c) Field dependence of the resistance value $R_{T_{m}}(H)$ where VLMT occurs. 
roughly fall on the same line, while it is in strong contrast with field dependence of resistance in the liquid phase. Summarized data of the activation energy in both solid and liquid phases are presented in Fig. 4(b). From the quantitative point of view, it seems that as vortex lattice is built up, the activation barrier in the solid phase abruptly increases by several times compared with the liquid phase. In the intermediate field region, from 200-600 Oe, a rather weak field dependence of the activation energy is observed in the solid phase, whereas $U$ monotonically decreases as field rises in liquid: $U(H)$ roughly follows a power law $U(H) \sim H^{\beta}$, where $\beta \approx-0.2$ for the solid state and $\approx-0.8$ for the liquid state. For higher fields, as periodic lattice transforms to the amorphous or glass vortex state, where random disorder determines the underlying vortex structure, the effective potential decreases sharper and a clear difference between solid and liquid states is lost: the value of $U$ becomes close to the one in the liquid phase. The low field region below 100 Oe was not considered because of a diverging value of the slope of $\ln \rho$ versus $1 / T$ in the zero field limit.

Last, what we are going to stress is concerning the field dependence of the resistance $R_{T_{m}}(H)$ where the VLMT occurs. In accordance with the current belief of field independence of the in-plane and out-of-plane resistivity jumps, Rojo et al. [25] presented an explanation based on the vortex cuttings and universalities of the structure factor at the freezing transition using the Verlet criterion [26] which is the counterpart of the Lindeman's criterion for the melting. This model predicts that $\rho\left(T_{m}\right) / \rho_{N} \sim$ $c_{L}^{2}\left(m_{a b} / m_{c}\right)^{1 / 2}$, where $\rho_{N}$ is normal state in-plane resistivity, $c_{L}$ is the Lindemann constant, and $m_{a b}$ and $m_{c}$ are the in-plane and out-of-plane effective masses, respectively. From our data we obtain $R\left(T_{m}\right) / R_{N} \sim 10^{-4}$ which gives the reasonable value of the anisotropy of the effective mass of $\gamma \sim 100-400$ by assuming the Lindemann constant in the range of 0.1-0.2. In contrast to the Rojo model, it is clearly seen in Fig. 4(c) that $R\left(T_{m}\right)$ depends on magnetic field: as the field is raised, the resistance jump shifts to lower levels of resistivity and roughly follows dependence $\ln R_{T_{m}} \sim-0.23 \times H$.

In summary, the vortex dynamics near VLMT in the single crystal $\mathrm{Bi}_{2} \mathrm{Sr}_{2} \mathrm{CaCu}_{2} \mathrm{O}_{8+\delta}$ was studied by precision in-plane resistance measurements. The VLMT in moving vortex states was observed to be very close to the one in the static vortex system. The estimated activation energy in the vortex solid phase strongly differs from one in the liquid state: in the lower field region $U$ depends weakly on magnetic field and increases with decreasing driving current density. We found that the VLMT coincides with the zero resistivity temperature in the zero current limit. As driving current is raised, the difference in resistance and activation energy between vortex solid and vortex liquid phases gradually disappears and resistance signature of the phase transition vanishes.
We gratefully acknowledge enlightening discussions with A.E. Koshelev, W. K. Kwok, G. W. Crabtree, V.M. Vinokur, D. T. Fuchs, and V. N. Zavaritsky.

*On leave from Faculty of Sciences, University of Montenegro, P.O. Box 211, Podgorica, Yugoslavia.

[1] A. E. Koshelev and V.M. Vinokur, Phys. Rev. Lett. 73, 3580 (1994).

[2] F. Nori, Science 271, 1373 (1996).

[3] S. Bhattacharya and M. J. Higgins, Phys. Rev. Lett. 70, 2617 (1993).

[4] U. Yaron et al., Nature (London) 376, 753 (1995).

[5] J. A. Fendrich et al., Phys. Rev. Lett. 77, 2073 (1996); G. W. Crabtree et al., J. Low Temp. Phys. 105, 1073 (1996).

[6] H. Pastoriza and P.H. Kes, Phys. Rev. Lett. 75, 3525 (1995).

[7] K. Kadowaki, Physica (Amsterdam) 263C, 164 (1996).

[8] S. Watauchi et al., Physica (Amsterdam) 259C, 373 (1996).

[9] D. T. Fuchs et al., Phys. Rev. B 54, R796 (1996).

[10] C. D. Keener et al., Phys. Rev. Lett. 78, 1118 (1997).

[11] T. Tsuboi, T. Hanaguri, and A. Maeda, Phys. Rev. B 55, R8709 (1997).

[12] H. Safar et al., Phys. Rev. Lett. 69, 824 (1992).

[13] W. K. Kwok et al., Phys. Rev. Lett. 69, 3370 (1992).

[14] T. Mochiku and K. Kadowaki, Trans. Mater. Res. Soc. Jpn. 19A, 349 (1993).

[15] N. Morozov et al., Phys. Rev. B 54, 3784 (1996).

[16] In principle, the activation energy $U$ changes as a function of temperature. But, since we do not know the exact functional form of activation energy $U=U(T, H, I)$, we extracted $U$ by the common approximation from the fit of the linear parts of $\ln R$ versus $1 / T$. The fall of the resistance data on the same curve only in the vortex solid when plotted as a function on $T-T_{m}$ (Fig. 4) indicates that the same simple conventional functional form like $U(T, H)=U_{o}(H)\left(1-T / T_{c}\right)^{a}$ cannot be applied on both vortex phases.

[17] T. Giamarshi and P. Le Doussal, Phys. Rev. B 55, 6577 (1997).

[18] M. Niderost et al., Phys. Rev. B 53, 9286 (1996).

[19] G. Blatter et al., Rev. Mod. Phys. 66, 1225 (1994).

[20] A. E. Koshelev, Physica (Amsterdam) 223C, 276 (1994).

[21] L. Burlachkov et al., Phys. Rev. B 54, 6750 (1996).

[22] D. T. Fuchs et al., Nature (London) 391, 371 (1998).

[23] J. Mirković et al. (to be published).

[24] After submission of our manuscript, two reports appeared in consistency with our results: A. Mazilu et al. [Phys. Rev. B 58, R8913 (1998)] discarded surface barriers from the experiment on Corbino geometry samples, while D. T. Fuchs et al. [Phys. Rev. Lett. 81, 3944 (1998)] reported agreement with Bragg-glass phase model [17] using the samples with contact geometry which probed the bulk properties avoiding surface effect.

[25] A. G. Rojo, A. K. Sood, and C. A. Balseiro, e-print condmat/9712017.

[26] J. P. Hansen and L. Verlet, Phys. Rev. 184, 159 (1969). 